[Agr. Biol. Chem., Vol. 33, No. 11, p. 1541 1552, 1969]

\title{
Bacteriophages of Clostridium saccharoperbutylacetonicum
}

\author{
Part XVII. The Structure of Phage HM 2 \\ By Seiya Ogata, Noriaki Nagao*, Zyun Hidaka* \\ and Motoyoshi Hongo \\ Laboratory of Applied Microbiology, Department of Agricultural Chemistry, \\ * Laboratory of Plant Pathology, Department of Agriculture, \\ Kyushu University, Fukuoka, Japan \\ Received March 24, 1969
}

\begin{abstract}
The fine structure of phage HM 2 (group I) active on Clostridium saccharoperbutylacetonicum was studied by an electron microscopy with a negative-staining technique, and compared with those of more conventional types, phages HM 3 (group II) and HM 7 (group III), whose tails were clearly observed by a shadow-casting technique. This study revealed that phage HM 2 had an intricate tail which was not observed by a shadow-casting technique.

Phage HM 2 has an icosahedral head about $450 \AA$ in diameter and a non-contractile tail about $300 \AA$ long. The distal $130 \AA$ of the tail axis has 1 width of $80 \AA$ which is wider than the upper portion of the tail ( 50 to $60 \AA$ ). The distal enlargement is not seen in the hollow tail. Twelve fibrous-shaped appendages are attached symmetrically at the upper portion of tail axis and extend toward the distal base of the tail. Their length is a little shorter than $300 \AA$. They combine with divalent cations in the phage dilution medium, and also adsorb the host cell debris.

Phage HM 3 has an icosahedral head about $770 \AA$ in diameter and a tail about $1000 \AA$ long and $150 \AA$ wide with contractile sheath. Phage HM 7 has an icosahedral head about $750 \AA$ in diameter and a long non-contractile tail about $2000 \AA$ long and about $120 \AA$ wide with forked tip.

The structure of the tail of phage HM 2 is quite different from those of phages HM 3 and HM 7 hitherto described and those of the various phages of other bacteria.
\end{abstract}

Phage HM 2 (group I) of $\mathrm{Cl}$. saccharoperbutylacetonicum showed the peculiar behaviors on exposure to deoxyribonuclease (DNase) in vitro, ${ }^{1,1}$ and in vivo, ${ }^{31}$ and to monovalent and divalent cations (or polyamines) in vitro. ${ }^{4,5}$ These behaviours were studied by the chemical and physical techniques. ${ }^{1251}$ It is also of value to study this particular phage by the electron microscopy.

1) M. Hongo and S. Ogata, Agr. Biol. Chem., 32, 459 (1968).

2) M. Hongo and S. Ogata, ibid., 33, 1 (1969).

3) M. Hongo and S. Ogata, ibid., 32, 1401 (1968).

4) M. Hongo, A. Murata and S. Ogata, ibid., 33, 331 (1969).

5) M. Hongo and S. Ogata, ibid., 33, 488 (1969).
An electron micrograph of a shadow-casting preparation of phage HM 2 was reported, ${ }^{6 !}$ but did not throw any light on the structure and its specific behaviours stated above. Also, little was known about the structure of the phages of Clostridium sp. (except Cl. perfringens). Therefore, in this paper, the fine structure of phage HM 2 and other phages have been examined by the electron microscopy of phage preparations stained with potassium phosphotungstate. The knowledge of fine structure of phage may provide further insight

6) M. Hongo and A. Murata, Agr. Biol. Chem., 30, 292 (1966). 
into the mechanism of the phage attachment to host and the injection of the deoxyribonucleic acid into host.

\section{MATERIALS AND METHODS}

Phages and their hosts. Phage HM 2 (group I) and phage HM 3 (group II) were grown on a strain N1-4. of Clostridium saccharoperbutylaetonicum,1,7) and phage HM 7 (group III) was grown on strain N1-504.7) A temperate phage $\mathrm{HM} \mathrm{T}$ was obtained from the lysogenic strain, Nl-4 (HM T). $\left.{ }^{8}\right)$

Preparation of phage. The phage lysates were purified and concentrated by four cycles of the differential centrifugation as described previously, 1,6) The phage pellet was then suspended in either distilled water or $0.005 \mathrm{M}$ Tris- $\mathrm{HCl}$ buffer ( $\mathrm{pH} 7.2$ ) unless otherwise specified.

Electron microscopy. The phage preparation was mixed with an equal volume of 1 to $2 \%(\mathrm{w} / \mathrm{v})$ potassium phosphotungstate ( $\left.\mathrm{P}^{r} \mathrm{TA}\right)(\mathrm{pH} 7.0)$, and placed on grids coated with collodion-carbon. Electron micrographs were taken by a JEM-7Y or JEM-5Y electron microscope (Japan Electron Optics Laboratory Co.).

\section{RESULTS}

\section{Morphology of phage HM 2}

The structure of Phage HM 2 is shown schematically in Fig. 1 and the negatively stained electron micrographs are presented in Figs. 2, 3, 4, 5, 6 and 7.

\section{1) Size and structure of head}

The head of phage HM 2 has a regular hexagonal outline and is about $450 \AA$ in diameter. The thickness of the head membrane of empty particle is about $15 \AA$. In Fig. 3 , the small head capsomeres can be seen, but their arrangement is not clear.

\section{2) Size and structure of the tail axis and the tail appendages}

The tail axis is clearly visible in the whole particles (Figs. 3 and 4) and in the empty particles (Figs. 3(F) and 5). In usual negatively stained preparations, however, the tail axis cannot be distinguished from the appendages which extend either downward parallel with the tail axis or divergently as far as the distal base of it. The tail axis is about $300 \AA$ long. The distal $130 \AA$ of the tail axis has a width of about $80 \AA$, and is wider than the upper portion of the tail axis (Figs. 3 and 4, arrow b). The tail of empty particle does not show the distal enlargement

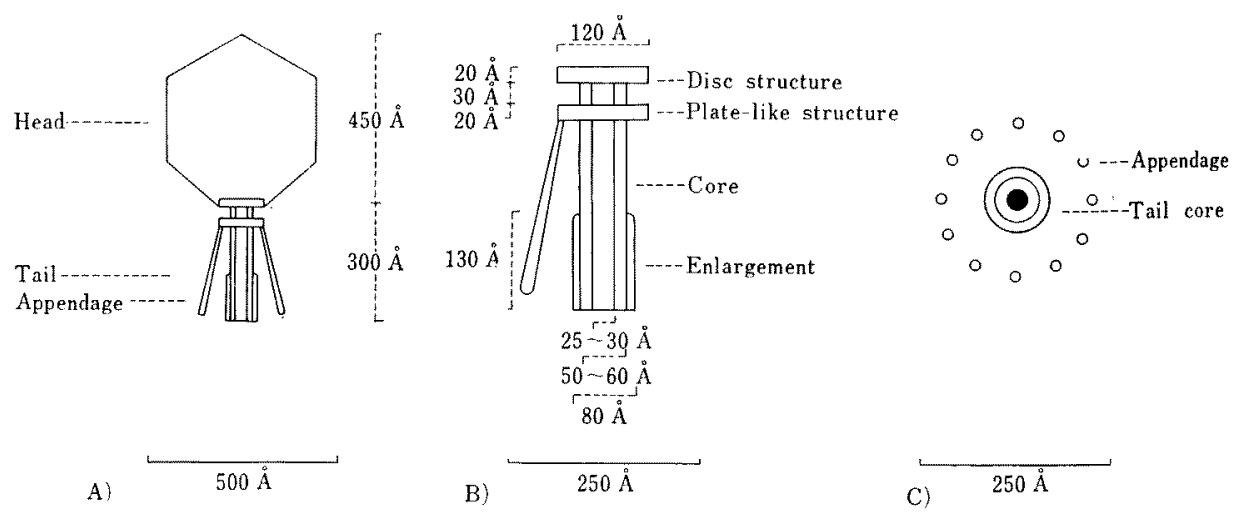

FIG. 1. Schematic Diagram of Phage HM 2.

A) Lateral view. B) Lateral view of detached tail. C) Distal view of tail.

7) M. Hongo and A. Murata, Agr. Biol. Chem., 29, $1149(1965)$.
8) M. Hongo, A. Murata and S. Ogata, Agr. Biol. Chem., 33, 337 (1969), 


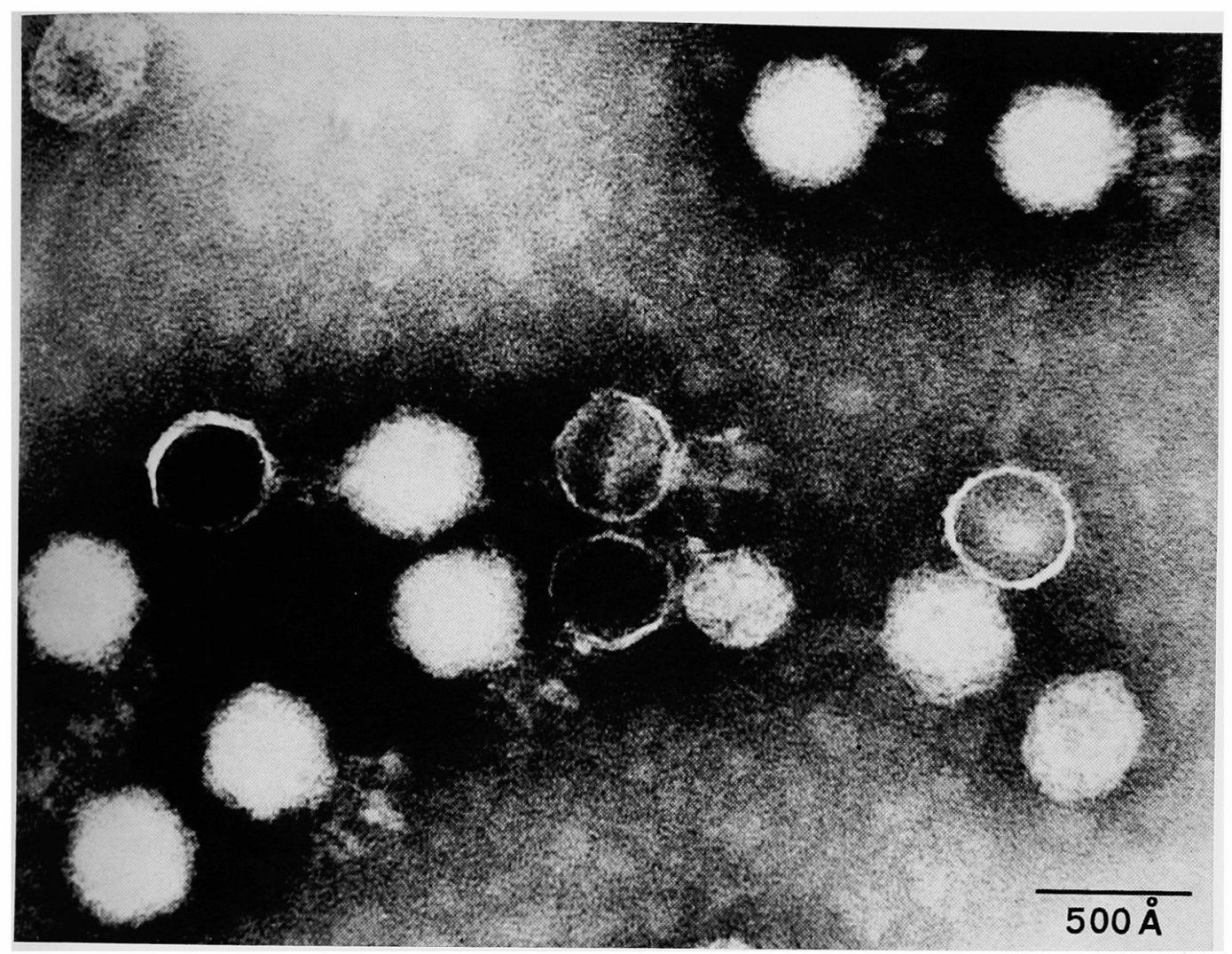

FIG. 2. Phage HM 2 Negatively Stained with PTA. $(\times 370,000)$

(arrow c). The width of the hollow tube of tail in several empty (Fig. $5(\mathrm{~A}, \mathrm{~B}, \mathrm{C})$ ) and partly empty particles (Fig. $3(\mathrm{~F})$ ) is 50 to $60 \AA$ in outside and 25 to $30 \AA$ in inside. In Fig. $5(F, G)$ the mannr in which the tail axis is attached to the head can be clearly seen; there is a disc at the point of attachment of the tail axis to the head (arrow d). In the detached tail in Fig. 6, this disc structure can be seen more clearly at the top of the core where it joines to the head membrane (Fig. 6(D, F)).

There is a plate-like structure just below the disc, which looks like a thin line or a collar (Figs. 3(A), 5(D) and 6, arrow a). In Fig. $6(\mathrm{~A}, \mathrm{~B}, \mathrm{C})$, the plate enlarges in dia- meter. This structure seems to be a part of the protein units of appendage (Figs. 6 and 7 ); in the latter figure, this structure and appendages are detached from the core.

Twelve fibrous-shaped appendages seem to be attached symmetrically to the plate-like structure and extend toward the distal base of the tail. Three to six of the appendages in most particles are distinct in lateral views. In a distal view of the tail, twelve appendages are clearly shown (Fig. 7). They are arranged radially around the tail axis which, in Fig. 7 (B, C, E), shows an opening of 25 to $30 \AA$ in the center of the core, but in Fig. 7 (A), shows a closed core. The width of appendage is not determined because of its 


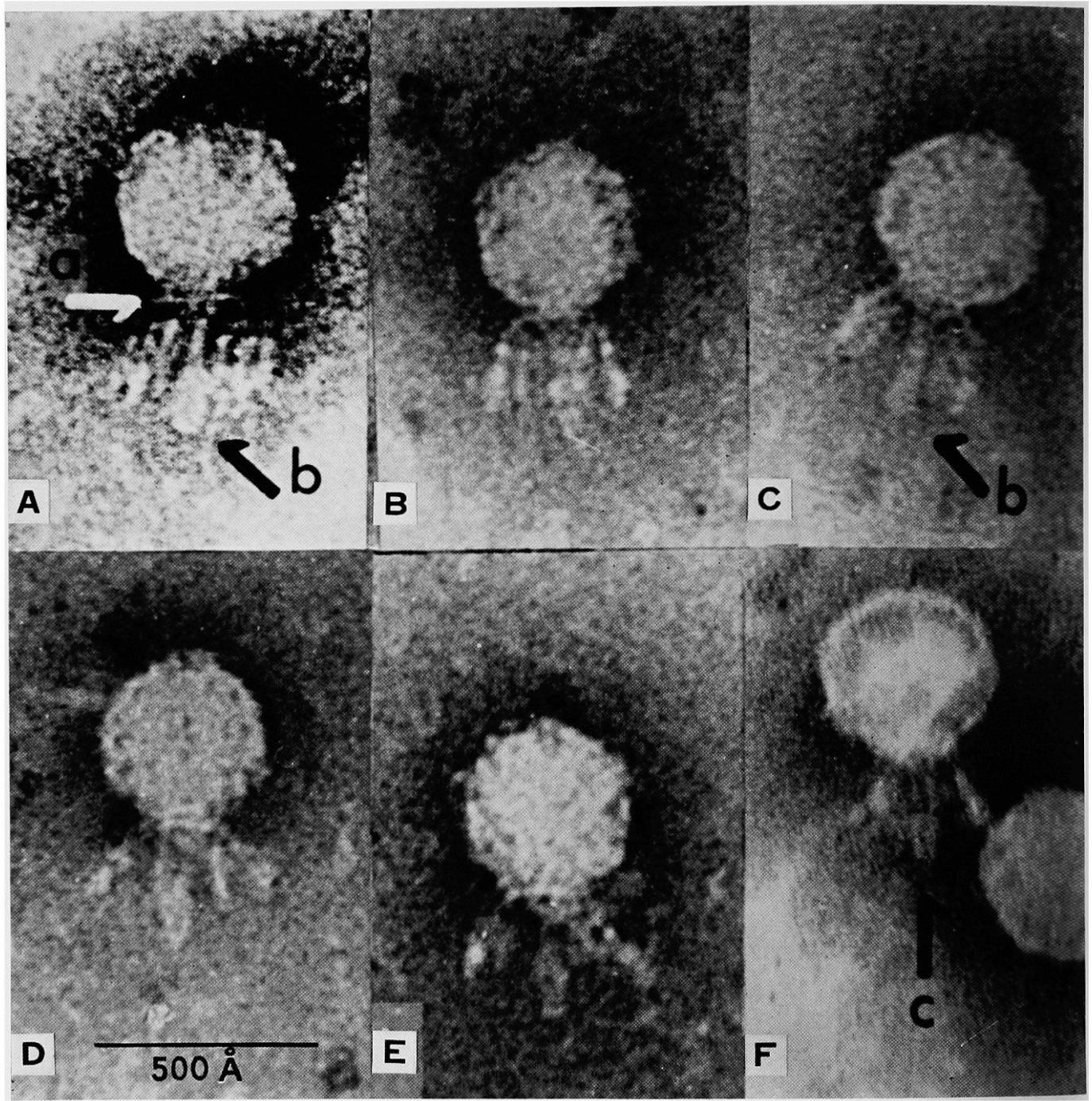

FIG. 3. Lateral View of Whole Particles and Partly Empty Particle of Phage HM 2. $(\times 690,000)$

thin structure. In Fig. 8, the appendages are shown attached to the cell debris. There is no evidence that the tail axis and appendages are contractile.

3) Morphology of phage HM 2 in lysate and in buffer containing $\mathrm{Mg}^{2+}$

The features stated above are of the prepa- ration of the purified phage in distilled water or dilute buffer. The appendages in these solutions are spread out like an unfolded fan, as mentioned above (especially in Fig. $4(\mathrm{H})$ ). On the other hand, the feature presented in Fig. 9 are of the crude preparation obtained from the supernatant $(5000 \times g, 15 \mathrm{~min})$ of a lysate of the phages. The appendages con- 


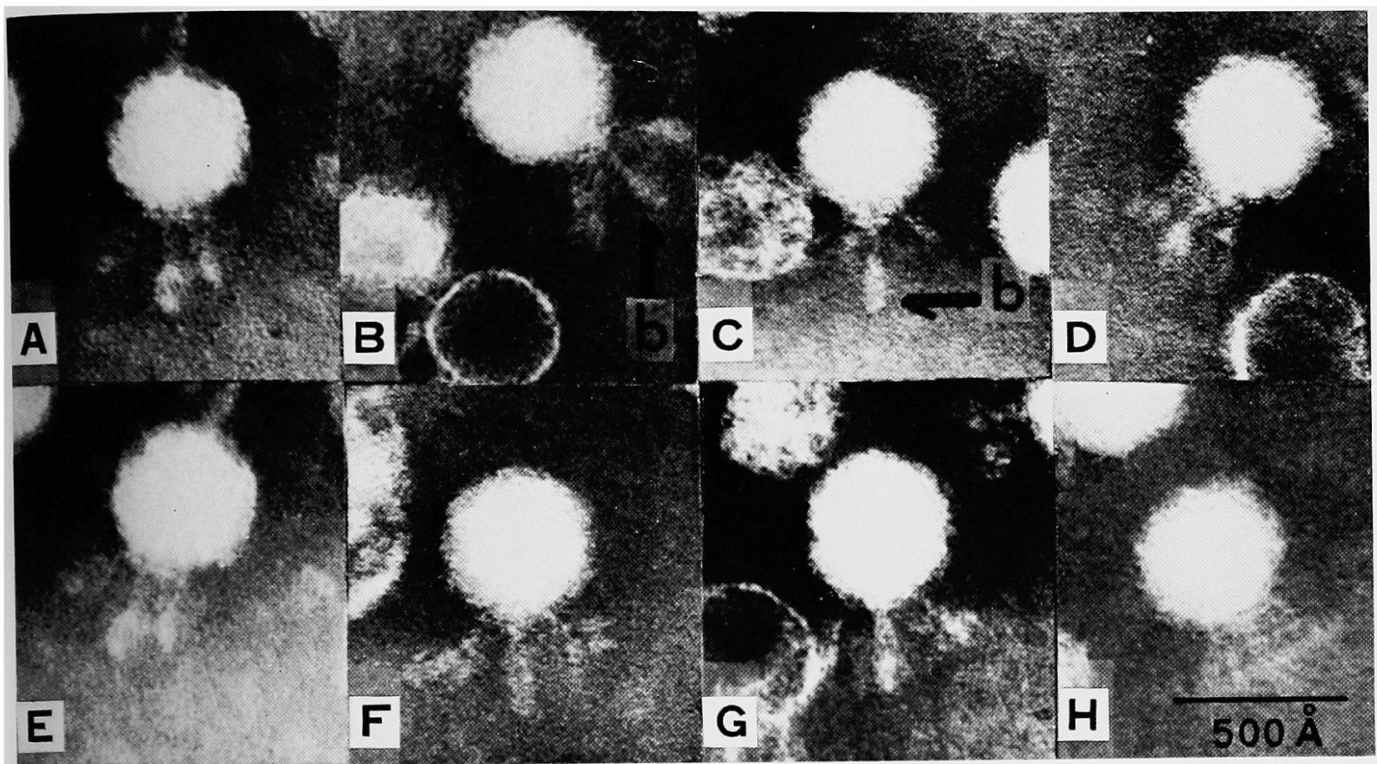

FIG. 4. Lateral View of Whole Particles of Phage HM 2. $(\times 370,000)$

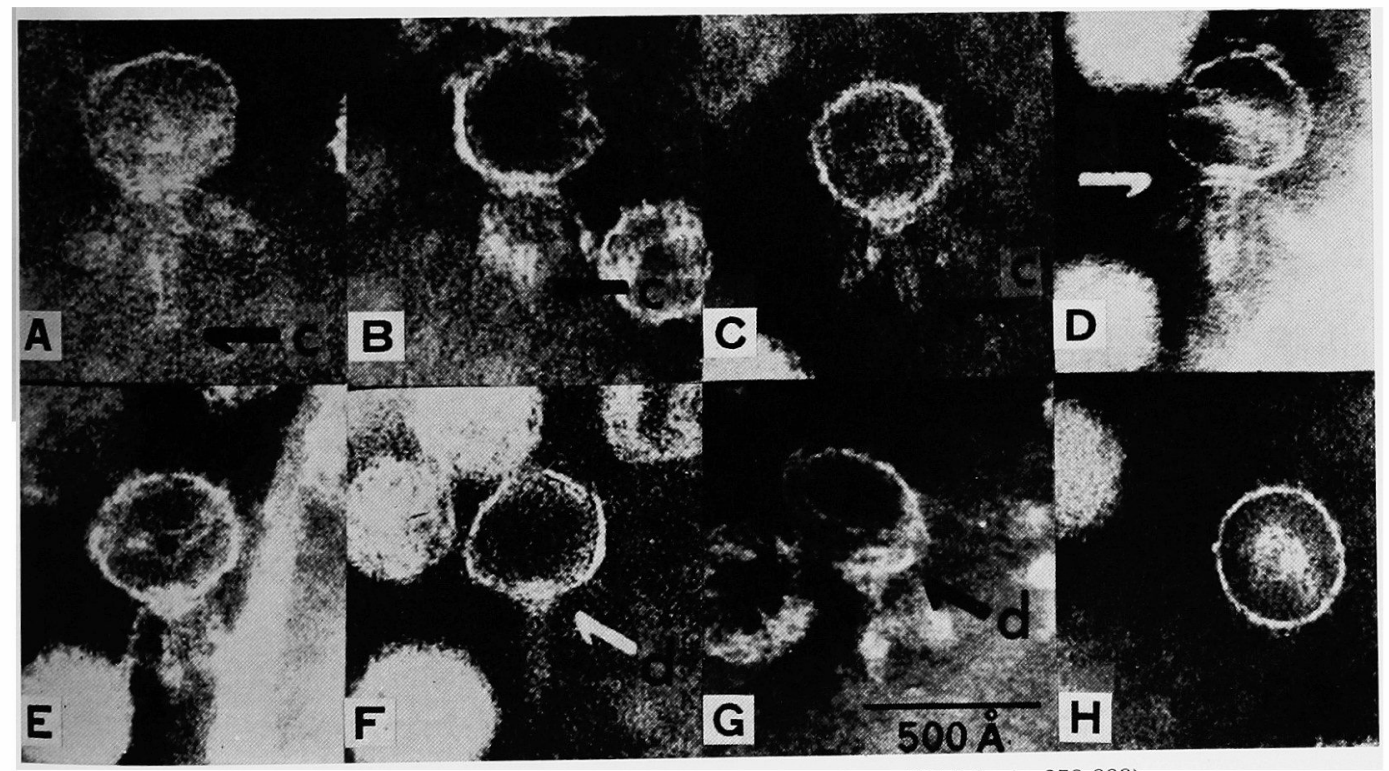

FIG. 5. Lateral View of Empty Particles of Phage HM 2. $(\times 370,000)$ 


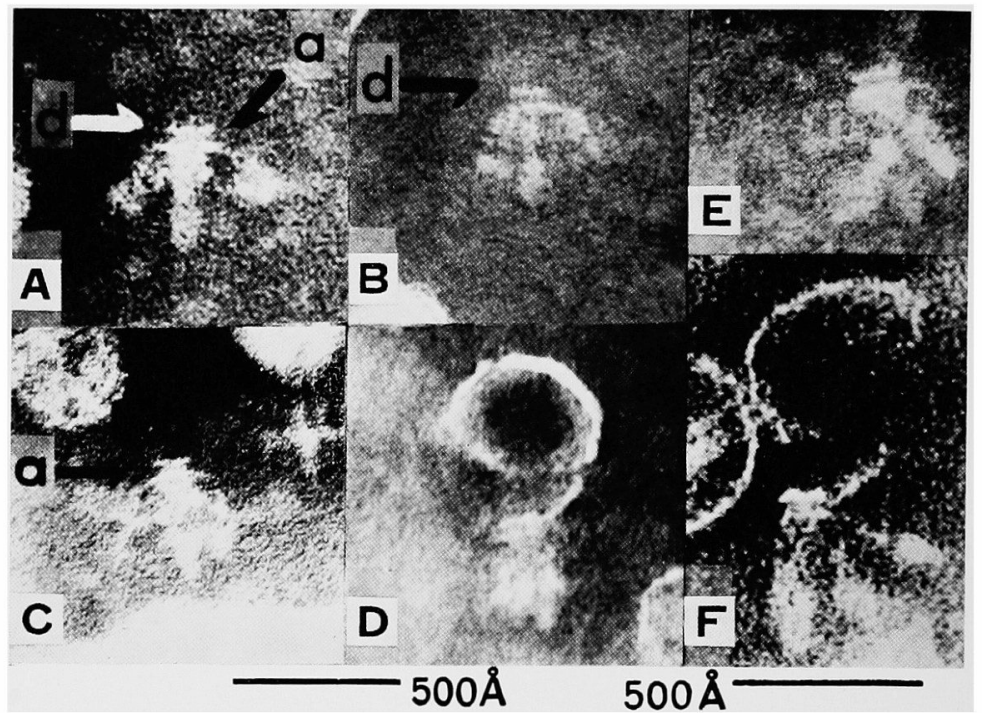

FIG. 6. Detached Tail of Phage HM 2 Showing Disc at the Top of the Tail and Plate-like Structure with Attached Appendages.

A, B, C and D) $(\times 350,000) ; \mathrm{E}, \mathrm{F})(\times 410,000)$

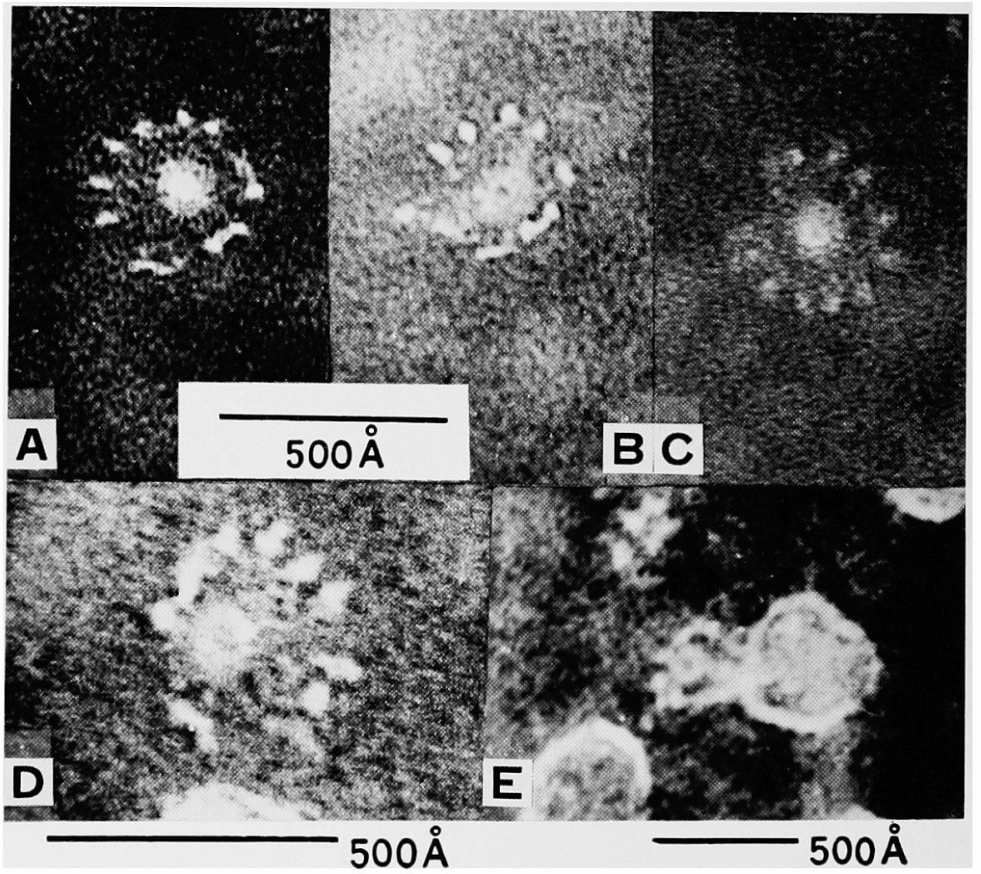

FIG. 7. Distal View of Tail.

A, B and C) Twelve appendages are shown $(\times 480,000)$

D) Plate-like structure with attached appendages $(\times 600,000)$

C) Empty particle prepared by DNase treatment $(\times 300,000)$ 


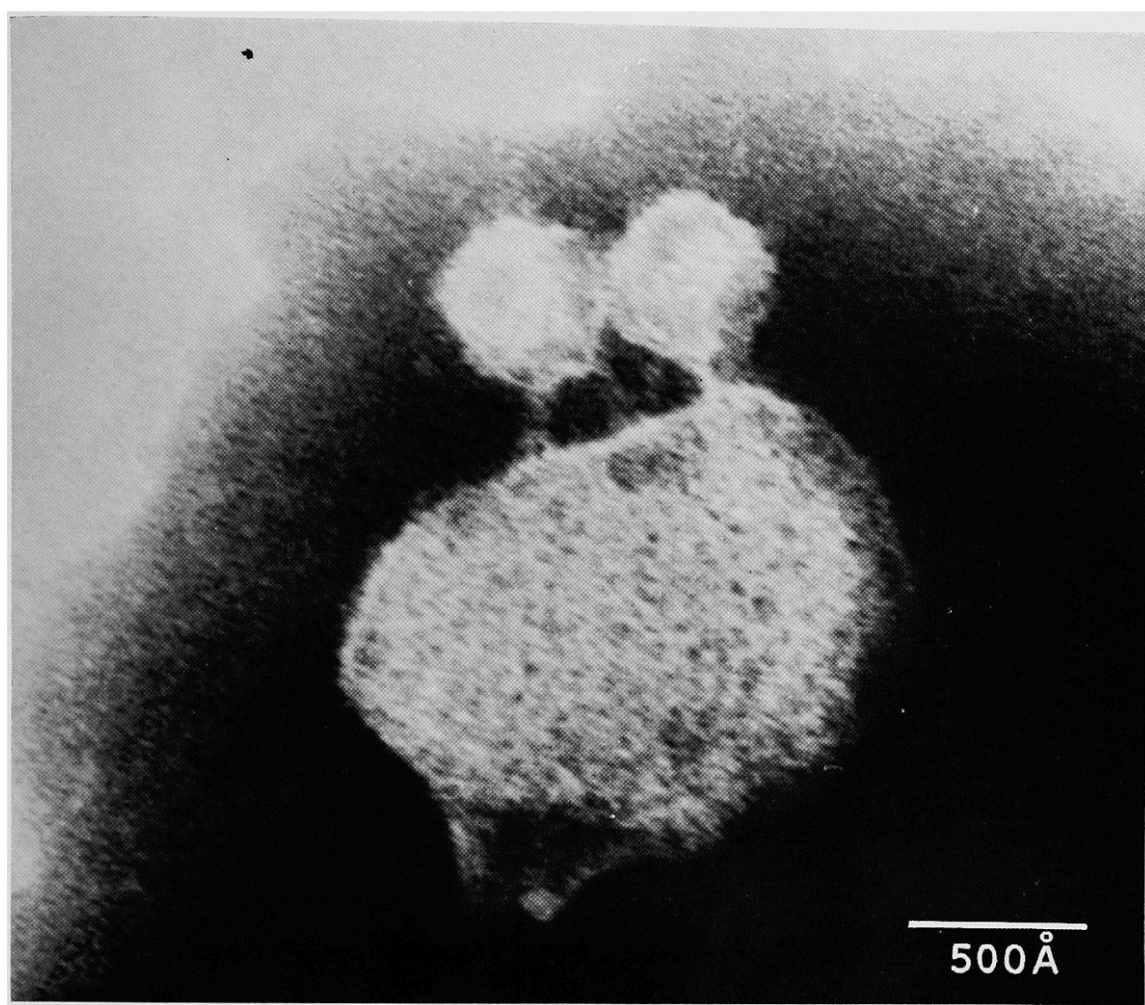

FIG. 8. Phage HM 2 Adsorbed to the Cell Debris. $(\times 390,000)$

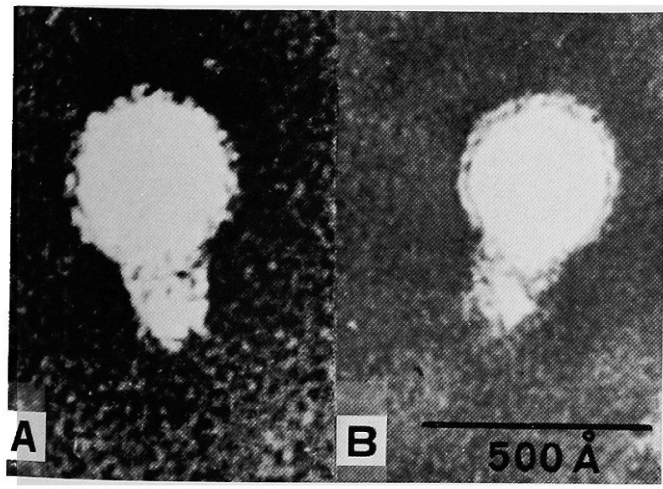

FIG. 9. Structure of Phage HM 2 in Lysate. $(\times 550,000)$

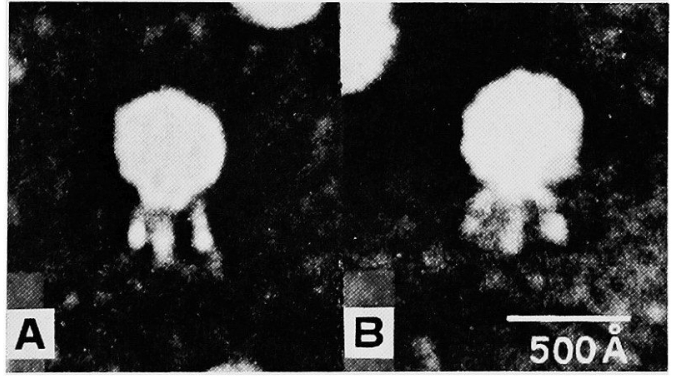

FIG. 10. Structure of Phage HM 2 in Buffer Containing $1 \times 10^{-3} \mathrm{M} \mathrm{Mg}^{2+} .(\times 260,000)$ 


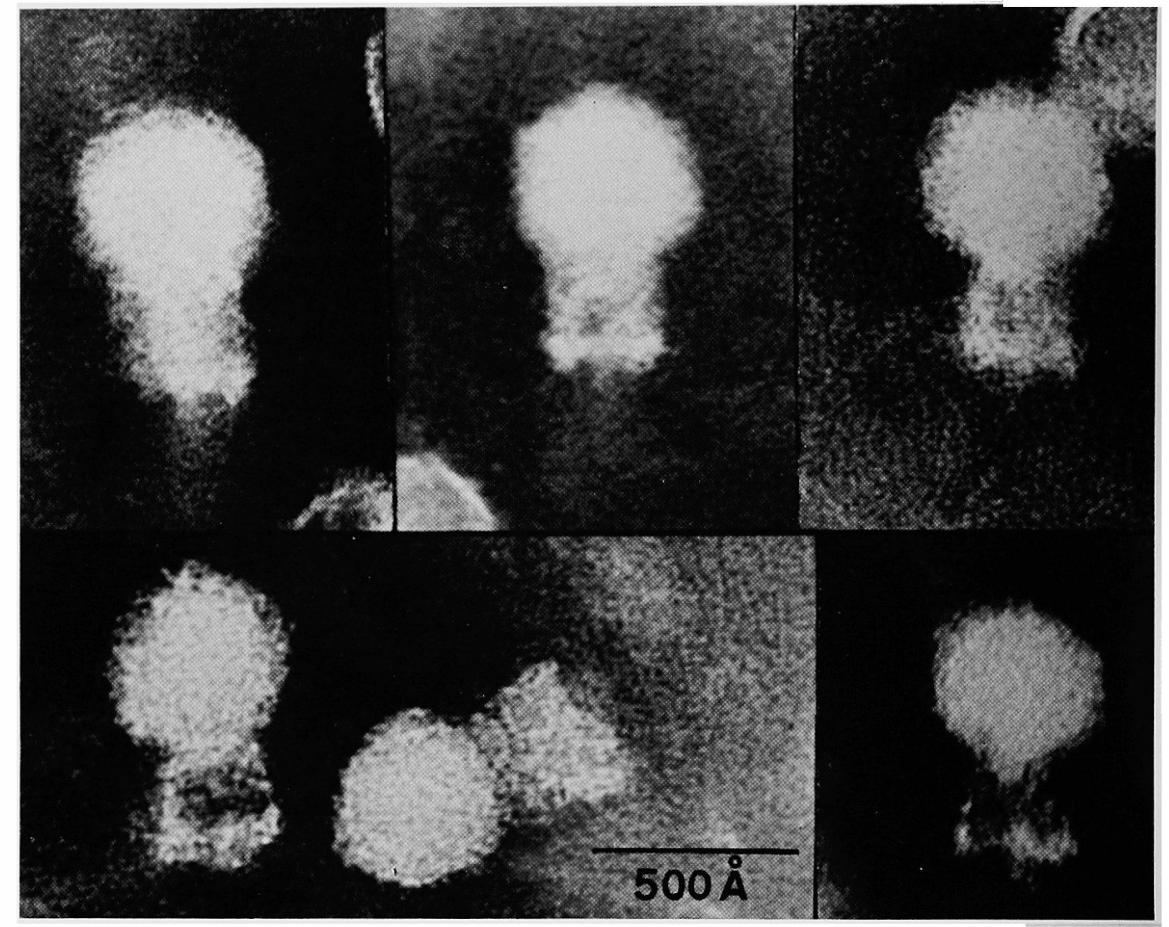

FIG. 11. Structure of Phage HM 2 in Buffer Containing $5 \times 10^{-3} \mathrm{M} \mathrm{Mg}^{2+} .(\times 430,000)$

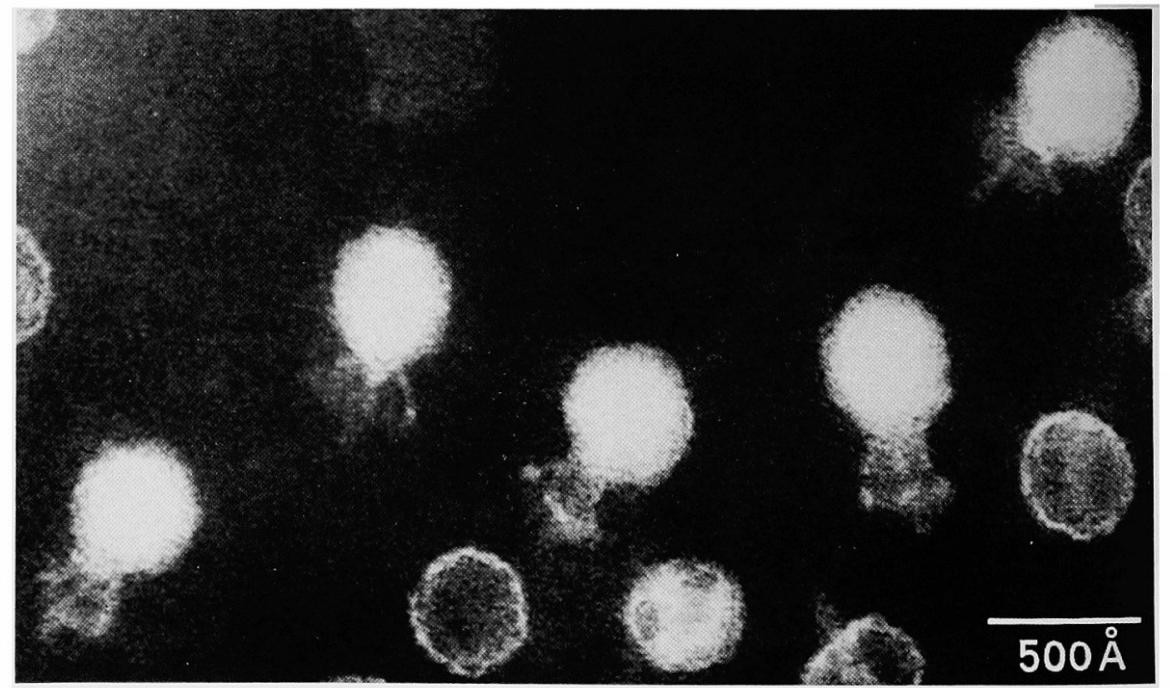

FIG. 12. Phage HM 2 from EDTA-treated Preparation. $(\times 310,000)$ 

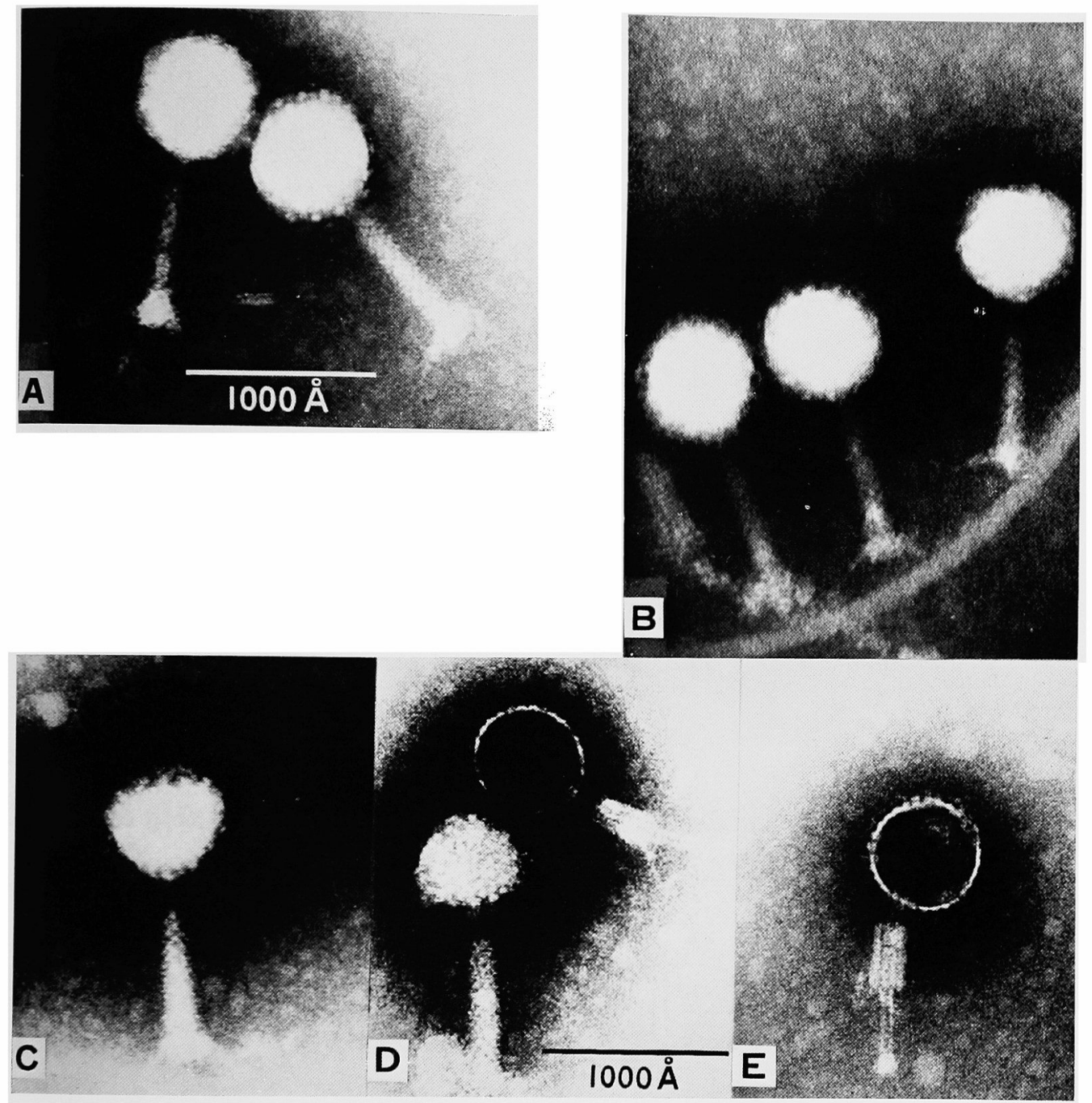

FIG. 13. Phage HM 3 Negatively Stained with PTA. $(\times 245,000)$

tract inward toward the tail axis and are tightly grouped. In Fig. 9(B), they adsorb the cell debris. These features are seen very frequently in the supernatant of suspension of confluently plaques, as well.

The purified phages were suspended in the buffer containing $1 \times 10^{-3} \mathrm{M}$ or $5 \times 10^{-3} \mathrm{M} \mathrm{Mg}^{2+}$ instead of water or the buffer alone and ob- served with electron microscope. The features are presented in Figs. 10 and 11, respectively. In Fig. 10, the appendages get together into three and four groups. In the presence of $5 \times 10^{-3} \mathrm{M} \mathrm{Mg}^{2+}$, they are so closely grouped that each appendage cannot be distinguished (Fig. 11).

In contrast, when the phages in the buffer 


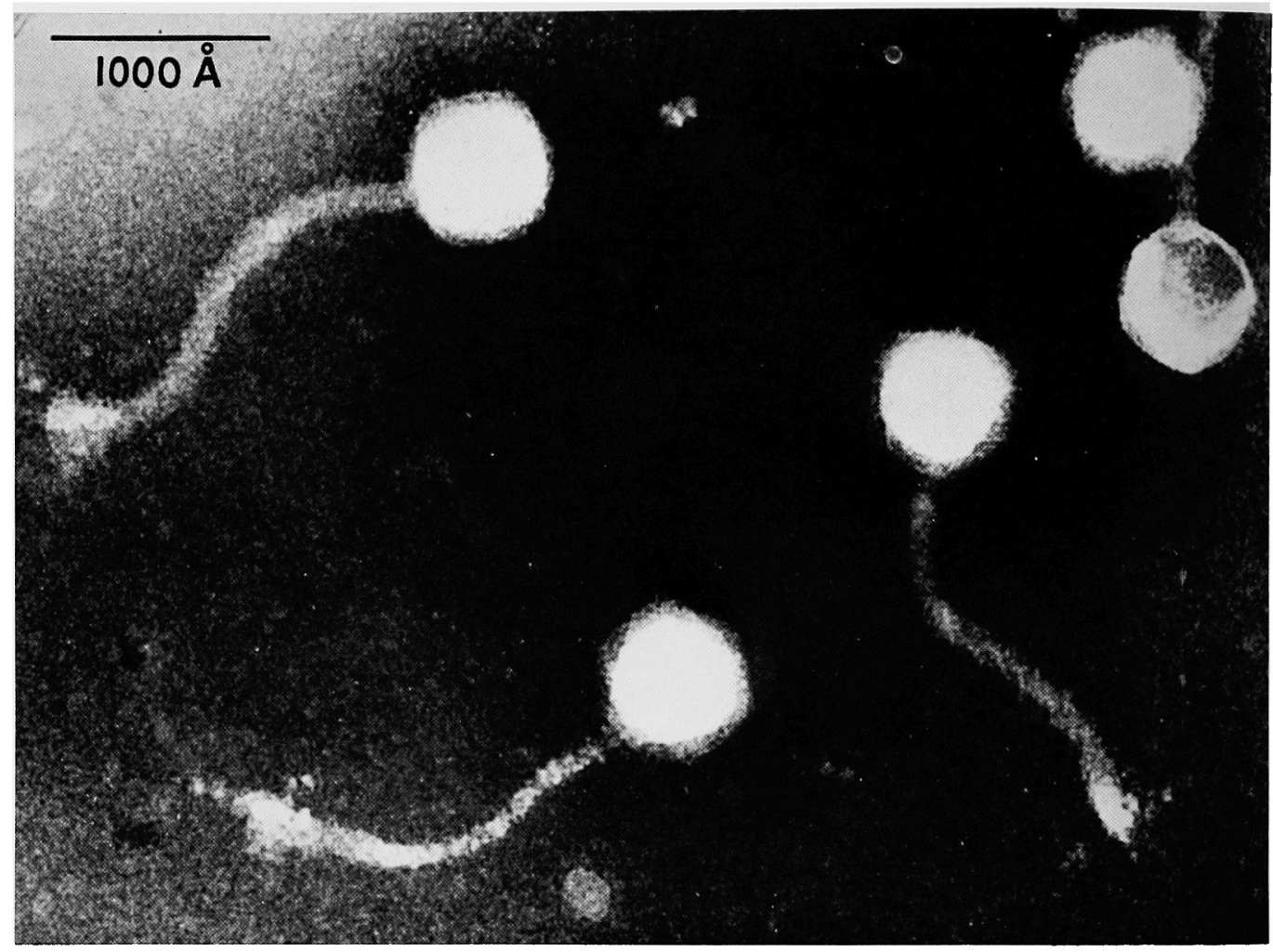

FIG. 14. Phage HM 7 Negatively Stained with PTA. $(\times 245,000)$

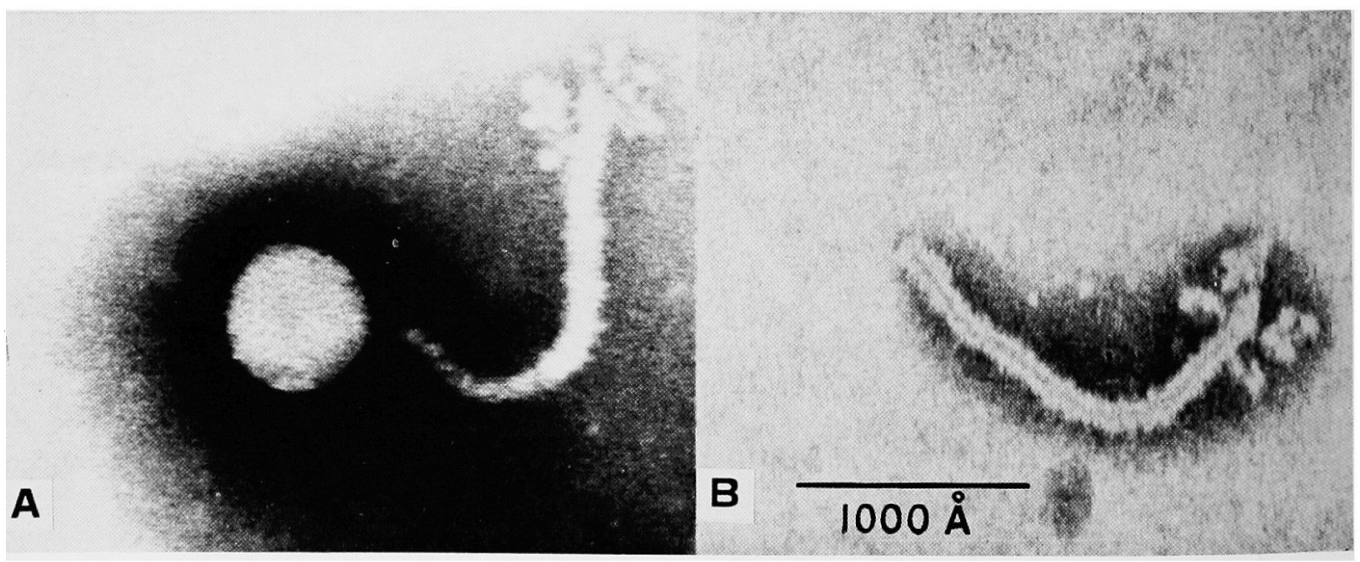

FIG. 15. Tail of Phage HM 7 with Forked Tip. $(\times 250,000)$ 
containing $\mathrm{Mg}^{2+}$ were treated with $5 \times 10^{-3} \mathrm{M}$ EDTA, according to Bradley and Kay, ${ }^{9}$ the individual appendage can be distinguished again (Fig. 12).

\section{Size and structure of phage $H M T$}

A temperate phage $H M T$ is similar to phage HM 2 in size and structure as well as in serology. ${ }^{81}$

\section{Size and structure of phage HM 3}

The structure of phage HM 3 is shown in Fig. 13. The head has a regular hexagonal outline about $770 \AA$ in diameter. The capsomers are clearly seen in side views, but their arrangement has not been determined. The tail is $1000 \AA$ long and has the typical appearance of a contractile type with striated sheath and tail fibres. The striated sheath is about $900 \AA$ long and $150 \AA$ wide. There are cross-striations with a spacing of about $30 \AA$. The number of tail fibres is difficult to be ascertained, but most likely from 3 to 6 . The empty phage in Fig. 13(E) shows clearly that the tail consists of a hollow core surrounded by a contractile sheath. The core is about $75 \AA$ wide; the inside width is about $40 \AA$. The contracted sheath is about $420 \AA$ long and $210 \AA$ wide.

\section{Size and structure of phage $H M 7$}

The structure of phage HM 7 is shown in Fig. 14. The head has a regular hexagonal outline about $750 \AA$ in diameter. The tail is a non-contractile and flexible and about $2000 \AA$ long and $120 \AA$ wide. Cross-striations are not clear even in empty tail, indicating that they are composed of tightly packed sub-units. In Fig. 15, the inner hollow tube of tail (about $30 \AA$ ) is seen. Some fibres are present at the tail tip.

9) D. E. Bradley and D. Kay, J. gen. Microbiol., 23, $553(1960)$.

\section{DISCUSSION}

Phage HM 2 (group I) is the most interesting in three phages described in this paper.

Though it belongs morphologically to the group of phage with a short and noncontractile tail, ${ }^{9}$ it possesses some interesting structural characteristics.

The features of phage HM 3 (group II) and phage HM 7 (group III) observed under electron microscopy with a shadow-casting technique $^{6 /}$ are very similar to those observed with a negative-staining technique. The overall length is about the same in both cases. Phage HM 3 is structurally similar to T-even phages $^{9,101}$ of $E$. coli and phage SP $8^{11}$ of $B$. subtilis. Phage HM 7 is structurally similar to phage $\mathrm{Fl}^{10}$ of $E$. coli and phage PN-1 of $B$. natto. ${ }^{121}$ On the other hand, the feature of phage HM 2 obtained by negtive-staining specimen is different from that obtained by shadow-casting specimen. ${ }^{61}$ The diameter of its head is found to be $450 \AA$ in the former but $1000 \AA$ in the latter. ${ }^{6}$ As the structure of the tail with 12 appendages is complicated and delicate, in the shadow-casting specimen the tail may be burried in a layer of Crmetal and it may be seen to be just a part of the head structure. Therefore, it was difficult to ascertain the presence or the shape of the tail. Phage HM 2 is structurally similar to phage $\phi 80^{13}$ and certain phages $(\mathrm{W}, \mathrm{WA}, \mathrm{Q})^{1 / 4}$ of $\mathrm{Cl}$. perfringens and phages GA-1 ${ }^{151}$ and $\phi 29^{16 t}$ of $B$. subtilis.

There is a lump of protein at the top of tail axis (Figs. 6 and $5(\mathrm{~F}, \mathrm{G})$ ), and the manner in which the tail is attached to the head can be clearly seen (Fig. 6(D, F)). The

10) D. E. Bradley, J. gen. Microbiol., 31, 435 (1963).

11) P. E. Davison, Virology, 21, 146 (1963).

12) M. Fujii, K. Oki, M. Makihara, Z. Kano and T. Takeya, Nippon Nogeikagaku Kaishi, 41, 39 (1967).

13) J. F. Vieu, A. Guelin and C. Dauguet, Ann. Inst. Pasteur, 109, 157 (1965).

14) S. Hirano and Y. Yonekura, Acta Med. Univ. Kagoshima., 9, 41 (1967).

15) D. E. Bradley, J. gen. Microbiol., 41, 233 (1965).

16) D. L. Anderson, D. D. Hickman and D. E. Reilly, J. Bacteriol., 91, 2081 (1966). 
similar structure has been observed within the empty particles of many phages with tail at the point of attachment of the tail to the head; this object was named disc or washer. ${ }^{15 \sim 17)}$ Bradley and $\mathrm{Kay}^{9}$ reported that there is probably similar disc within the head membrane as well as on the tail axis, preventing the tail from detaching. In case of phage $\phi 29$, a similar object was named an upper collar.

The distal portion of the tail axis of whole particle is wider than the axis of the upper portion of the tail. However, the axis of empty particle does not show the distal en" largement. This evidence may suggest that the roles of the distal enlargement of tail are similar to those of the contractile sheath of $\mathrm{T}$-even phage during attachment of the phage to the host cell and the injection of its DNA into the host.

There is a plate-like structure or collar just below the disc. Twelve appendages seem to be attached to this structure. It is not yet clear whether this structure is composed of a part of the protein units of appendage or another protein units; according to Figs. 6 and 7 (D), the former may be true. In case of phage $\phi 29$, the similar structure to which 12 appendages were attached was named a lower collar.

The appendages of phage HM 2 resemble those of phage $\phi 29$ in numbers and structure, though they are narrower than those of phage $\phi 29$ and less in the ratio of the length of the tail axis to the length of appendage than that of phage $\phi 29$. Phage GA-1 has six appendages. The shape of appendages of phage HM 2 seems to resemble the shape of the contractile sheath of certain phage (W, WA, Q) of $\mathrm{Cl}$. perfringens, but the appendages of phage HM 2 are not sheath and there is no indication that they are contratile. Phage

17) D. E. Bradley, J. gen. Microbiol, 35, 471 (1964).
HM 2 is often attached to the host cell and the cell debris at the distal portion of the appendages and tail axis (Figs. 8 and $9(\mathrm{~B})$ ). It is considered that the appendages are organella for adsorption to the host cell.

The feature in Fig. 9 shows the most native structure, because this is seen frequently in broth culture. The appendages are so closely grouped that the individual appendage cannot be distinguished in this cultural solution. In the buffer containing $\mathrm{Mg}^{2+}$, they became three or four groups (Fig. 10) or are closely grouped (Fig. 11). On the other hand, in water or dilute buffer, they are not grouped but are spread out like an unfolded fan (Figs. 2, 3 and 4). These evidences indicate that divalent cation such as $\mathrm{Mg}^{2+}$ and other polycationic agents are easily adsorbed by the appendages. By EDTA-treatment, the $\mathrm{Mg}^{2+}$ adsorbed are easily removed (Fig. 12).

The individual appendage (Figs. 3 and 4) or the plate-like structure (Figs. 3(A, B, D, E) and $4(\mathrm{~A}, \mathrm{~B})$ ) is shown in some cases. Thus, the structure of phage HM 2 is changed by a variety of components in the dilution medium; the molecular organizations of the phage became visible by the structural change. According to the previous reports, ${ }^{4,5}$ divalent cation such as $\mathrm{Mg}^{2+}$ and polyvalent cation strengthened the molecular structure of protein and DNA-protein complex of the phage and somehow prevented the destruction of its native structure. The closely packed structure observed in Figs. 9, 10 and 11 would be the structure stabilized and strengthened by $\mathrm{Mg}^{2+}$ and other cationic agents. On the other hand, according to another previous report, "phage HM 2 in the buffer containing $\mathrm{Mg}^{2+}$ was strongly inactivated by DNase, but in the lysate it was hardly inactivated. Therefore, the changes in the molecular structure of protein and DNA-protein complex of the phage in Figs. 10 and 11 are greater than in Fig. 9. This structural change would allow the penetration of DNase into site of action. ${ }^{3}$ 